\title{
Passive Radar based on the Specific Sequence of Wireless Network
}

\author{
Qian Zhao, Hui Zhao, etc \\ $\left\{\right.$ qzhao $^{1}$, hzhao $\left.{ }^{2}\right\} @$ bupt.edu.cn \\ Wireless Signal Processing and Networks (WSPN) Lab , \\ Key Laboratory of Universal Wireless Communications, Ministry of Education, \\ Beijing University of Posts and Telecommunications (BUPT), Beijing, 100876, China
}

\begin{abstract}
Passive radar locates the UAV target by processing the reflected signal from non-collaborative illuminators of opportunity (IOs). It will inevitably be affected by the direct-path interference and multipath interference. The conventional passive radar uses the reference channel to receive the direct-path signal as the reference signal for interference cancellation, which is complicated in hardware and susceptible to noise. However, most of the wireless signals carry the prior information used for synchronization or channel estimation, such as the preamble of WiFi signal or the CRS of LTE signal. This paper presents a passive radar system based on the specific sequence in the wireless network, which is the prior information of IOs. Firstly, we analyze the ambiguity function of several typical specific sequences and prove their feasibility as the reference signal. Then we adopt a coherent approach for interference cancellation. The system availability is proved by simulation. A $9.64 \mathrm{~dB}$ reduction of interference is observed.
\end{abstract}

Keywords: Unmanned Aerial Vehicles (UAVs) localization, interference cancellation, passive radar, wireless network.

\section{Introduction}

Unmanned aerial vehicle (UAV) applications are rapidly expanding [1], but they also bring numerous challenges and security risks. In some sensitive areas, it is particularly important to quickly detect and locate the UAVs [2]. Common active radars detect targets by emitting radar signals themselves, but it costs a lot and not suitable for large scale applications. Passive radar [3] detects and locates the target by processing reflected signals from non-collaborative illuminators of opportunity (IOs). It spends less by utilizing the existing communication equipments, and works in covert operation. In a word, it is more suitable for positioning the non-cooperative UAV.

In a conventional passive radar system[4], the receiver uses two receiving channels, in which one for surveillance and the other for reference. The antenna of the reference channel points to the IO and receives the direct wave signal from the IO; the antenna of the surveillance channel points to the sky and receives the target echo signal. Both channels are susceptible to noise while receiving valid signals. At the same time, the target echo signal in the surveillance channel will also be subject to interference such as the strong direct-path signal and the multipath signals. J. Tong [5] completed a detailed study on joint estimation of target position and velocity in the passive radar system. The CRLB of the target position depends not only on the IO waveform parameters, but also on the values of SNR and INR (interference-to-noise). 
Many broadcast and communication signals have been considered and analyzed as the possible passive radar sources, including FM radio, DVB-T, GSM, LTE and WiFi [6][7][8]. These wireless signals usually carry signals used for channel estimation or synchronization, which can be considered as prior knowledge at the receiver. For example, the Long-Term Evolution (LTE) needs cell specific reference signals (CRS) for channel estimation and correlation demodulation. And the $802.11 \mathrm{n} \mathrm{WiFi}$ standard specifies the preamble part for frame synchronization. Different from the existing passive radar solutions, this paper will use the sequence carrying prior knowledge in the IO signal as the reference signal. So the receiver can only use a single channel to receive the reflected signal from IOs. It can also avoid the delay and noise impact caused by the reference channel.

On the other hand, affected by the sidelobes of the surveillance antenna, direct path interference (DPI) and multipath interference (MPI) are often too higher than the target echo to accurately detect UAV target in passive radar systems[9]. If the interference is not eliminated, it is impossible to detect the target only by the conventional processing methods such as specifying the antenna direction and pulse compression[10]. Therefore, interference cancellation becomes a key issue in passive radar systems. At present, adaptive algorithms for interference cancellation mainly include: Least Mean Square (LMS), Recursive Least Squares (RLS), Extended Cancellation Algorithm (ECA)[11][12], Sequential Cancellation Algorithm (SCA), and so on[13]. The ECA has been shown to be effective against typical scenarios with a limited number of iterations.

In this paper, we propose a passive radar system based on a wireless network. The specific sequence in non-service signal is used as the reference signal, which is known to both transmitter and receiver. Firstly, we analyze the ambiguity function[14], and it is proved that the specific sequence carrying prior information is sufficient to replace the reference signal received by the reference channel. Then, the interference subspace matrix generated by the specific sequence filters the surveillance signal for interference cancellation. After cancellation, the time delay and frequency offset estimates of the UAV target can be obtained by the Range-Doppler processing [15] of the target echo signal. Therefore, the target position can be estimated through the classic time difference-of-arrival algorithm.

The paper is organized as follows: Section 2 analyzes the ambiguity functions of CRS in LTE and preamble in WiFi. Section 3 introduces the passive radar system model based on wireless networks. Section 4 derives an algorithm for extended interference cancellation. Simulation and analysis are discussed in section 5 , in the scenarios which parameters were generated by Winprop propagation model software. Finally, conclusions are drawn in section 6 .

\section{Ambiguity function analysis}

The sequence carrying prior knowledge in wireless network signals has a very specific structure designed for channel estimation or synchronization. It is called the specific signal in this paper. A brief analysis of the relevant factors influencing a passive radar system is provided here.

Range resolution and Doppler resolution are important parameters used in radar systems to indicate the ability to distinguish two or more targets. Range resolution $\Delta R$ can be written as:

$$
\Delta R=\frac{c}{2 B \cos (\beta / 2)}
$$


where $c, B$ and $\beta$ are the speed of light, the bandwith of the signal and the bistatic angle separately.The length of the pulse $T$ determines the Doppler resolution for that single pulse, which can be written as:

$$
\Delta f_{d}=\frac{1}{T}
$$

In this section, we analyze the ambiguity function of the 801.11n WiFi signal and the LTE signal separately. Considering $\beta=45^{\circ}$ and $T=0.05 \mathrm{~s}$, Tables. 1 shows parameters of these two IO signals in theory.

Table 1. Parameters of transmitted signals.

\begin{tabular}{lll}
\hline Parameters & $802.11 \mathrm{n}$ & LTE \\
\hline Bandwidth $B(\mathrm{~Hz})$ & $20 \mathrm{M}$ & $10 \mathrm{M}$ \\
Subcarrier spacing $\Delta f(\mathrm{~Hz})$ & $312.5 \mathrm{k}$ & $15 \mathrm{k}$ \\
sampling frequency $f_{s}(\mathrm{~Hz})$ & $40 \mathrm{M}$ & $15.36 \mathrm{M}$ \\
range resolution $\Delta R(\mathrm{~m})$ & 7.5 & 9.76 \\
Doppler resolution $\Delta f_{d}(\mathrm{~Hz})$ & 20 & 20 \\
\hline
\end{tabular}

Characteristics determined by the nature of the transmitted waveform in Tables.1 can be evaluated by ambiguity function in simulation:

$$
\left|\xi\left(\tau, f_{d}\right)\right|=\left|\int_{-\infty}^{\infty} s^{*}(t+\tau) s(t) e^{j 2 \pi f_{d} t} d t\right|
$$

where $\left|\xi\left(\tau, f_{d}\right)\right|$ is the value of ambiguity function of the transmitted signal $s(t), \tau$ denotes the delay, $f_{d}$ is the Doppler frequency, and $s^{*}(t)$ is the complex conjugate of $s(t)$. In practice, the ambiguity function is often digitally implemented on a computer and the equation becomes:

$$
\left|\xi\left(\tau, f_{d}\right)\right|=\left|\sum_{n=0}^{N-1} s[n] s^{*}[n+\tau] e^{j 2 \pi f_{d} n T_{s}}\right|
$$

where $s[n]=s\left(n T_{s}\right), T_{s}$ is the sample period and $N$ is the number of samples in the integration time.

A simulated ambiguity function of the preamble in the 802.11n signal is shown in Figure 1(a). As can be seen, the ambiguity function has a relatively high peak of $25.05 \mathrm{~dB}$ at the origin. Figure 1(b) shows the range profile $\left(f_{d}=0\right)$ of the ambiguity function, where the sidelobe levels are $21.41 \mathrm{~dB}$ and the range ambiguity is $0.8 \mu \mathrm{s}$. The corresponding distance is $1.2 \mathrm{~km}$. Other range ambiguities are multiple of $0.8 \mu \mathrm{s}$, which don't make much sense because the corresponding distances are beyond radar coverage. Figure 1(c) shows the Doppler profile $(\tau=0)$ of the ambiguity function, where the sidelobe levels are 8.66dB. 


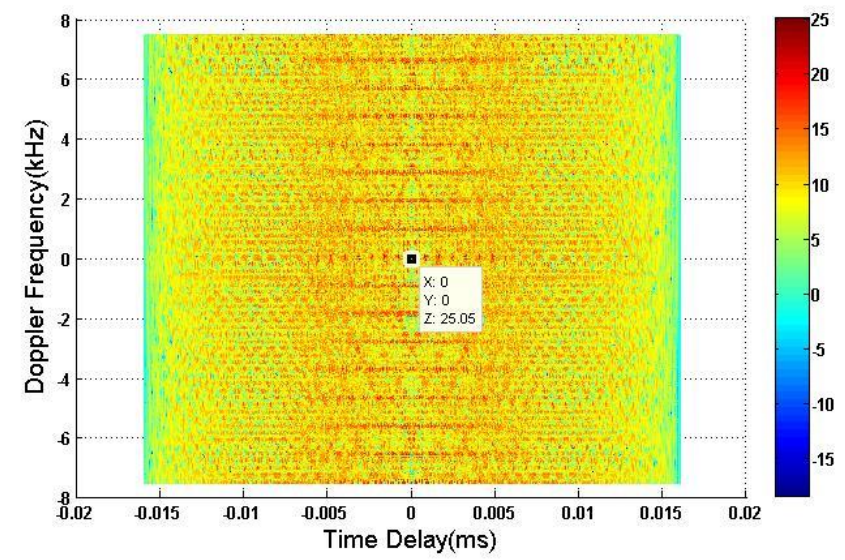

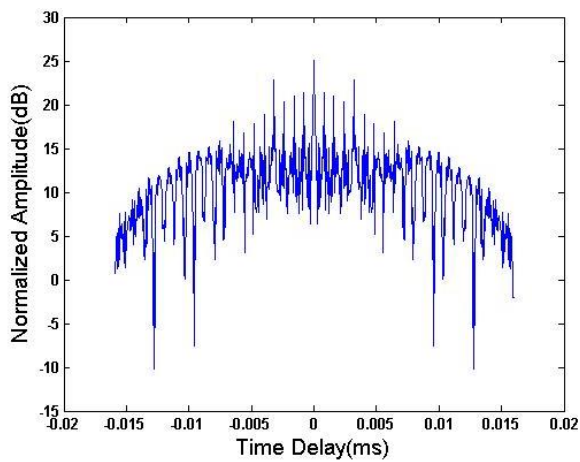

(b) (a)

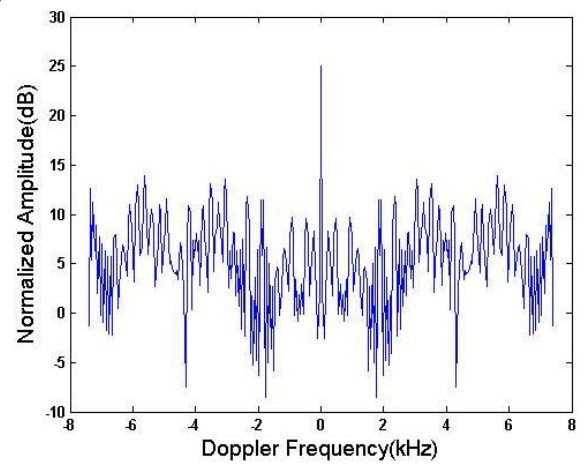

(c)

Fig. 1. (a) Ambiguity function of the preamble in $802.11 \mathrm{n}$ signal (b) Range profile ( $f_{d}=0$ ) (c) Doppler $\operatorname{profile}(\tau=0)$.

Similarly, the ambiguity function of the CRS in LTE signal is shown in Figure 2(a). The ambiguity function has a relatively high peak of $26.11 \mathrm{~dB}$ at the origin. Figure 2 (b) shows the range profile $\left(f_{d}=0\right)$ of the ambiguity function, where the sidelobe levels are $20.2 \mathrm{~dB}$ and the range ambiguity is $0.39 \mu \mathrm{s}$. The corresponding distance is $0.58 \mathrm{~km}$. Figure $1(\mathbf{c})$ shows the Doppler profile ( $\tau=0$ ) of the ambiguity function, where the sidelobe levels are $19.63 \mathrm{~dB}$.

Through the above description and discussion, the ambiguity caused by the sidelobe in the ambiguity function will not affect the positioning in a certain small range $(<500 \mathrm{~m})$. This paper proves the feasibility of using WiFi preamble and CRS signal as passive radar non-cooperative IOs, and estimates the detection capabilities of passive radar systems based on the wireless network. 


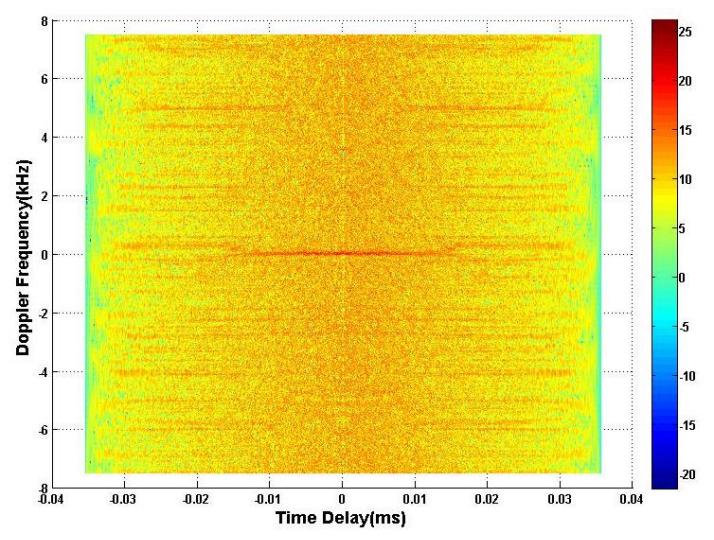

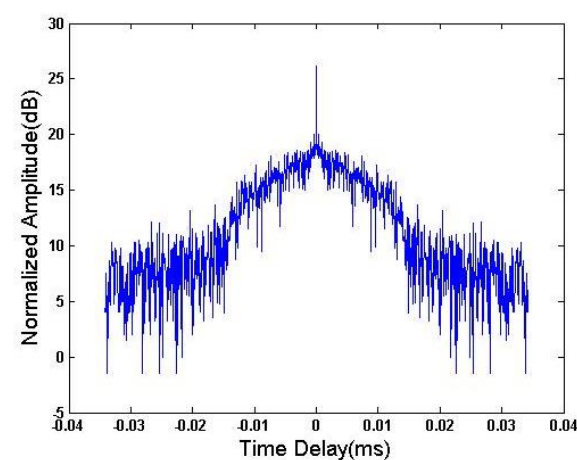

(b)

(a)

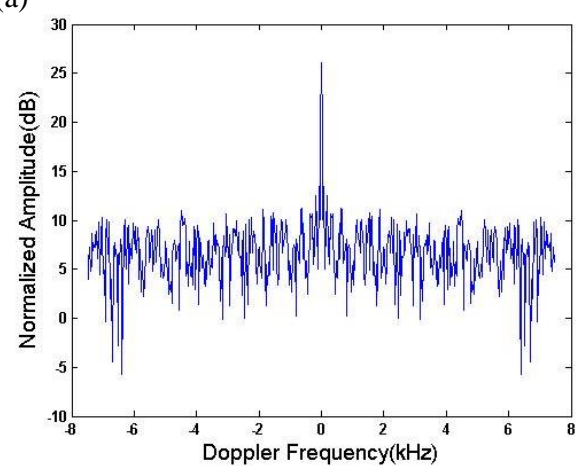

(c)

Fig. 2. (a) Ambiguity function of the CRS in LTE signal (b) Range profile ( $f_{d}=0$ ) (c) Doppler profile $(\tau=0)$.

\section{System model}

We consider the signal model in a typical passive radar geometry. The signal collected from the echo channel contains not only the target echo signal, but also DPI, MPI and noise signal. While the reference channel of conventional radar also contains the direct-path signal and noise signal. In this paper, the specific sequence with prior information was seen as the reference signal. 


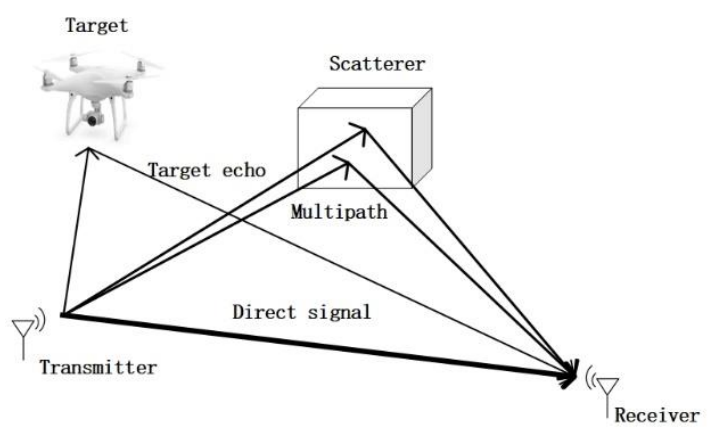

Fig. 3. Signal model.

As seen in Figure 3, assuming that multipath echoes can be expressed as the collection of the signal reflected by multiple fixed positions, the complex envelope of the signal at the receiver is given by:

$$
s_{R}(t)=A_{R} d(t)+\sum_{i=1}^{N c} c_{i}(t) d\left(t-\tau_{c i}\right)+\sum_{j=1}^{M} \alpha_{j} d\left(t-\tau_{j}\right) e^{j 2 \pi f_{d j} t}+n_{R}(t)
$$

where $A_{R} d(t), \sum_{i=1}^{N c} c_{i}(t) d\left(t-\tau_{c i}\right)$ and $\sum_{j=1}^{M} \alpha_{j} d\left(t-\tau_{j}\right) e^{j 2 \pi f_{d j} t}$ denote the direct-path signal, the stationary scatterers and the target echos respectively. $d(t)$ and $A_{R}$ are the complex envelope and complex amplitude of the direct-path signal; $c_{i}(t)$ and $\tau_{c i}$ are the complex amplitude and the delay of the i-th stationary scatterer $\left(i=1,2, \ldots, N_{C}\right), N_{C}$ is the number of scatterers; $\alpha_{j}, \tau_{j}$ and $f_{d j}$ are the complex amplitude, the delay (with respect to the direct-path signal) and the Doppler frequency of the $\mathrm{j}$-th target $j=1,2, \ldots, M, M$ is the number of targets and the value is one in this paper; $n_{R}(t)$ is the thermal noise contribution at the receiver.

The complex amplitude $c_{i}(t)$ are considered slowly varying functions of time, so that they can be represented by only a few frequency components around zero Doppler:

$$
c_{i}(t)=c_{i} e^{j 2 \pi f_{c t} t}
$$

where $f_{c t}$ and $c_{i}$ are the Doppler shift and complex amplitude of the i-th multipath echo.

Assuming operation with a sampling frequency $f_{s}$ which satisfies the Nyquist theorem, the samples $s_{R}$ collected at the surveillance channel at time instants $t_{i}=i / f_{s}=i T_{s}, i=0, \ldots, N-1$, are arranged in an $N \times 1$ vector:

$$
s_{R}=\left[s_{R}[0], s_{R}[1], s_{R}[2], \cdots, s_{R}[N-1]\right]^{T}
$$

Similarly,we obtain $N$ samples of the reference signal in the following vector:

$$
s_{\text {ref }}=\left[s_{\text {ref }}[-R+1], \cdots, s_{\text {ref }}[0], \cdots, s_{\text {ref }}[N-1]\right]^{T}
$$

where $R$ is the extra simple points to ensure direct path in $s_{R}$ and $s_{\text {ref }}$ line up exactly.

The detection process in passive radar is based on the evaluation of the delay-Doppler crosscorrelation function $(\mathrm{CCF})$ between the surveillance and the reference signal: 


$$
\xi(l, p)=\sum_{i=0}^{N-1} s_{R}[i] \cdot s_{r e f}^{*}[i-l] e^{-\frac{j 2 \pi p i}{N}}
$$

where $l=0, \ldots, R-1$ is the time bin representing the time delay $\tau[l]=l T_{s} . p$ is the Doppler bin representing the Doppler frequency $f_{d}[p]=p /\left(N T_{s}\right)$

While in the Delay-Doppler cross-correlation function of the received signal $s_{R}$ and the reference signal, peak of target echo is masked by the sidelobes of the direct-path signal, so it is necessary to pay attention to the cancellation of interference in practice.

\section{Interference cancellation algorithm}

An effective cancellation filter for passive radar can be obtained by an Least Square approach. The algorithm searches for a minimum residual signal power after cancellation of the interference (direct-path signal and multipath echoes), thus:

$$
\min _{\alpha}\left\{\left\|s_{R}-\mathbf{X} \alpha\right\|^{2}\right\}
$$

where $\mathbf{X}$ is the interference subspace matrix and $\alpha$ is the adaptive coefficient.

$$
X=B\left[\Lambda_{-p} S_{\text {ref }} \ldots \Lambda_{-1} S_{\text {ref }} S_{\text {ref }} \Lambda_{1} S_{\text {ref }} \ldots \Lambda_{\mathrm{p}} S_{\text {ref }}\right]
$$

where $\mathbf{B}$ is an incidence matrix that selects only the last $\mathrm{N}$ rows of the following matrix:

$$
\mathbf{B}=\left\{b_{i j} \mid i \in[1, N], j \in[1, N+R-1]\right\} \quad b_{i j}=\left\{\begin{array}{cc}
1 & i=j-R+1 \\
0 & \text { otherwise }
\end{array}\right.
$$

$\boldsymbol{\Lambda}_{\mathbf{p}}$ is a diagonal matrix that applies the phase shift corresponding to the $\mathrm{p}$-th Doppler bin:

$$
\boldsymbol{\Lambda}_{\mathbf{p}}=\left[\begin{array}{cccc}
1 & 0 & \cdots & 0 \\
0 & e^{j 2 \pi p} & \cdots & 0 \\
\vdots & \vdots & \ddots & \vdots \\
0 & 0 & \cdots & e^{j 2 \pi p(N+R-1)}
\end{array}\right]
$$

$\mathbf{S}_{\text {ref }}$ is a matrix whose columns are the zero-Doppler, delayed versions of the reference signal $s_{r e f}$ :

$$
\mathbf{S}_{\text {ref }}=\left[s_{\text {ref }} \mathbf{D} s_{\text {ref }} \mathbf{D}^{2} s_{\text {ref }} \cdots \mathbf{D}^{K-1} s_{\text {ref }}\right]
$$

D is a $0 / 1$ permutation matrix that applies a delay of a single sample and is defined as:

$$
\mathbf{D}=\left\{d_{i j} \mid i, j \in[1, N+R-1]\right\} \quad d_{i j}=\left\{\begin{array}{cc}
1 & i=j+1 \\
0 & \text { otherwise }
\end{array}\right.
$$

The columns of matrix $\mathbf{X}$ in (10) define a basis for an M-dimensional interference subspace, where $M=(2 P+1) K$. Solving (10) yields:

$$
\alpha=\left(\mathbf{X}^{H} \mathbf{X}\right)^{-1} \mathbf{X}^{H} s_{R}
$$

Therefore, the surveillance signal after cancellation becomes:

$$
\hat{s}_{t}=s_{R}-\mathbf{X} \alpha=\left[I-\mathbf{X}\left(\mathbf{X}^{H} \mathbf{X}\right)^{-1} \mathbf{X}^{H}\right] s_{R}=\mathbf{P} s_{R}
$$


where the projection matrix $\mathbf{P}$ projects the received vector $s_{R}$ in the subspace orthogonal to the interference subspace.

\section{Simulation and analysis}

In order to evaluate the performance of the passive radar system mentioned above, we consider a study case in the open filed of suburb without the dense buildings. A $802.11 \mathrm{n} \mathrm{WiFi}$ transmitter is used as IO with a bandwidth of $20 \mathrm{MHz}$. The transmit power of the transmitter is $0.1 \mathrm{~W}$. The noise spectral density $N_{0}$ is set as $-101 \mathrm{dBm}$. Based on the transmission distance of the 802.11n WiFi signal in a suburb area, the distance between the transmitter and the receiver is set to $300 \mathrm{~m}$. Assuming that the speed of the UAV target is $50 \mathrm{Km} / \mathrm{h}$, and the flight direction is consistent with the direction of the direct wave signal from the transmitter, the value of the Doppler frequency is calculated to be $111 \mathrm{~Hz}$. The propagation modeling software WinProp provides a more realistic simulation: depending on the scenario, predictions are based on topographical, clutter, and building databases; different transmission modes can be defined and the coverage maps are computed individually for each transmission mode.

WinProp outputs the time delay, Doppler frequency and power information of each path in the scene. Considering the UAV target is in $70 \mathrm{~m}$ height, the parameters are shown in Tables.2.

Table 2. Parameters of received signals ( $70 \mathrm{~m}$ height).

\begin{tabular}{lcccc}
\hline Signal & Direct-path & Multipath echo1 & Multipath echo2 & Taget echo \\
\hline Time Delay $(\mathrm{ns})$ & 1003.97 & 1056.44 & 1055.89 & 1101.17 \\
Doppler frequency $(\mathrm{Hz})$ & 0 & 0 & 0.5 & 111 \\
Power $(\mathrm{dBm})$ & -84.48 & -88.69 & -88.36 & -98.18 \\
\hline
\end{tabular}

Firstly, considering the sampling frequency at receiver is $40 \mathrm{MHz}$, we assume that cancel range $\mathrm{K}=4$ and Doppler area $\mathrm{p}=100$. Then we apply the interference cancellation algorithm mentioned in Section 4. The delay-Doppler cross-correlation function between the surveillance and the reference signal was shown in Figure.4.
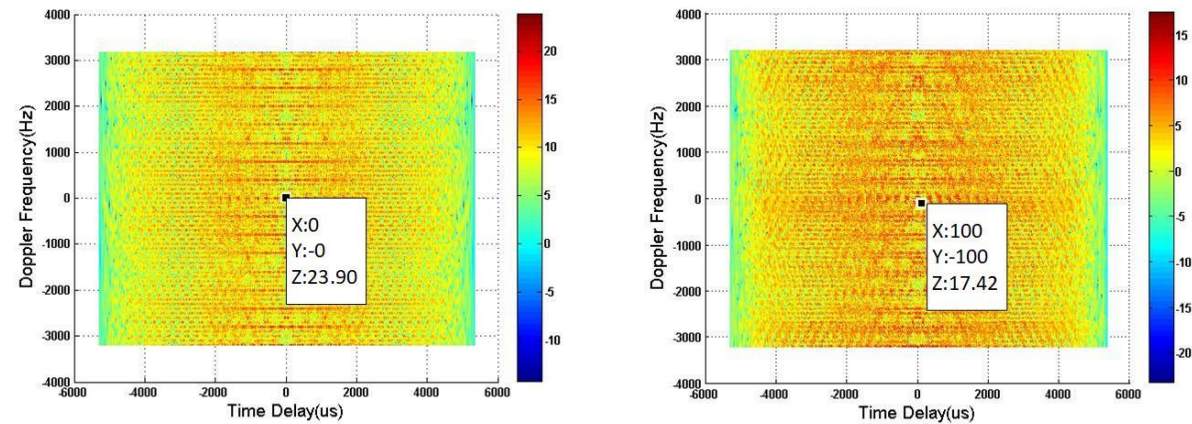

Fig. 4. (a) CCF before cancellation (b) CCF after cancellation.

Figure.4(a) shows the CCF output of the surveillance signal and the reference signal before cancellation. And Figure.4(b) shows the CCF output after removing the direct-path interference 
and all multipath interferences laying in the first $K$ range bins $(K=4$ and $P=100)$. Here, the weak UAV target now appears as a strong peak. In Figure.4(b), the location of the weak target is shown obviously. The most prominent peak represents the target echo whose amplitude is $17.42 \mathrm{~dB}$. At origin, the value of the function is about $14.26 \mathrm{~dB}$, which means that the amplitude of the direct-path signal was eliminated more than $9.64 \mathrm{~dB}$. After cancellation, the amplitude of the direct-path signal is $3.16 \mathrm{~dB}$ less than the target echo. Then the UAV target can simply be detected by using a simple constant false alarm rate (CFAR) detector such as the CA-CFAR detector.

Here, the peak to average power ratio (PAPR) tests are introduced for evaluating the performance of the algorithm. For calculating the PAPR, a 50m height UAV target echo and the multipath echo are introduced with characteristics tabulated in Tables.3.

Table 3. Parameters of received signals (50m height).

\begin{tabular}{lccc}
\hline Signal & Direct-path & Multipath echo & Taget echo \\
\hline Time Delay $(\mathrm{ns})$ & 1003.97 & 1022.78 & 1053.41 \\
Doppler frequency $(\mathrm{Hz})$ & 0 & 0 & 111 \\
Power $(\mathrm{dBm})$ & -84.48 & -87.56 & -96.00 \\
\hline
\end{tabular}

We analyze the performance of the algorithm, assuming that cancel range $K=4$ and Doppler area $\mathrm{p}=100$.

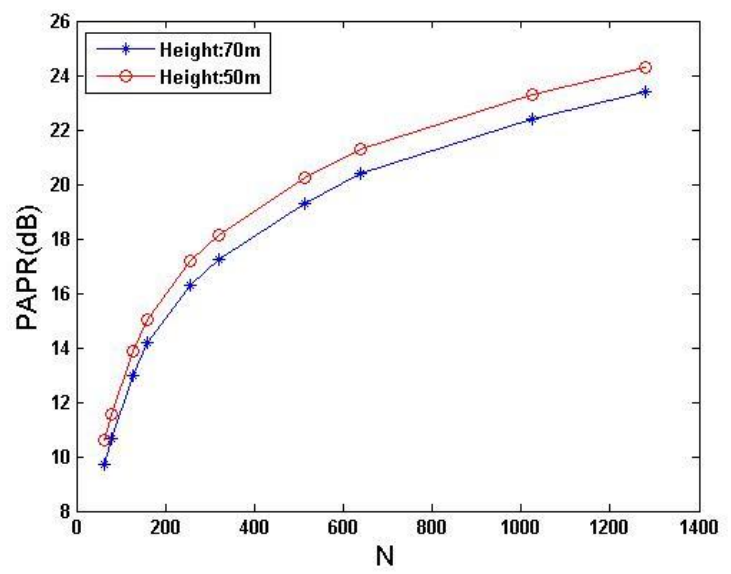

Fig.5. Diagram of PAPR versus the number of IO signal samples.

In Figure.5, a simulated PAPR curve of the interference cancellation algorithm versus the number of IO signal samples is shown in the comparison of different height. As can be seen, the amplitude of the target after cancellation will be increased by increasing the number of samples, and reduced by increasing the height. This is consistent with the Cramér-Rao lower bound analysis. One can observe that there exists a significant performance degradation when the IO signal samples is under 256. 


\section{Conclusion}

For UAV localization, this paper proposed a passive radar system based on the specific sequence of the wireless network. Ambiguity function valued the detection performance of the specific sequence with prior information. The cancellation algorithm was based on the projections of the received signals in a subspace orthogonal to the interference. Using the specific sequence as the reference signal makes the system more economical and effective. By using the propagation model generated by Winprop, the simulation results showed that the approach counteracted interference more effectively. It is of practical significance to detect UAV targets by using this radar system in the future. 
Acknowledgments. This work was supported in part by the Beijing Municipal Science and Technology Commission Research under Project(Z181100003218015).

\section{References}

[1] Shakhatreh H, Sawalmeh A, Al-Fuqaha A, et al. : Unmanned aerial vehicles (uavs): A survey on civil applications and key research challenges. IEEE Access. vol. 7, pp. 48 572-48 634 (2019)

[2] Z. Jingcheng, F. Xinru, Y. Zongkai, and X. Fengtong.: Uav detection and identification in the internet of things. 15th International Wireless Communications \&amp; Mobile Computing Conference (IWCMC).IEEE. pp. 1499-1503 (2019)

[3] H. Griffiths.: From a different perspective: principles, practice and potential of bistatic radar. 2003 Proceedings of the International Conference on Radar (IEEE Cat. No. 03EX695). pp. 1-7 (2003)

[4] P. Howland.: Passive radar systems. IEE Proceedings-Radar, Sonar and Navigation.vol. 152, no. 3, pp. 105-106 (2005)

[5] J. Tong, H. Gaoming, T. Wei, and P. Huafu.: Cramér-rao lower bound analysis for stochastic model based target parameter estimation in multistatic passive radar with direct-path interference. IEEE Access. vol. 7, pp. 106 761-106 772 (2019)

[6] Bartoletti, Stefania, A. Conti, and M. Z. Win.: Passive radar via LTE signals of opportunity. IEEE International Conference on Communications Workshops IEEE(2014)

[7] Palmer, James E.:DVB-T Passive Radar Signal Processing. IEEE Transactions on Signal Processing 61.8,pp.2116-2126 (2013)

[8] Q Zheng, K Zheng, H Zhang, VCM Leung,: Delay-optimal virtualized radio resource scheduling in software-defined vehicular networks via stochastic learning, IEEE Transactions on Vehicular Technology,65 (10), pp. 7857-7867, (2016)

[9] J. Chen, L. Xu, W. Zhang, and W. Wu.: An effective interference cancellation algorithm in noncooperative passive radar. IEEE 13th International Conference on Signal Processing (ICSP). pp. 1480 1484 (2016)

[10] M. Meller.: Cheap cancellation of strong echoes for digital passive and noise radars. IEEE Transactions on Signal Processing. vol. 60, no. 5, pp. 2654-2659 (2012)

[11] F. Colone, R. Cardinali, and P. Lombardo.: Cancellation of clutter and multipath in passive radar using a sequential approach. IEEE Conference on Radar. pp. 7-pp (2006)

[12] W. Feng, J.-M. Friedt, G. Cherniak, Z. Hu, and M. Sato.: Direct path interference suppression for short-range passive bistatic synthetic aperture radar imaging based on atomic norm minimisation and vandermonde decomposition. IET Radar, Sonar \& Navigation (2019)

[13] R. Cardinali, F. Colone, C. Ferretti, and P. Lombardo.: Comparison of clutter and multipath cancellation techniques for passive radar. IEEE Radar Conference. pp. 469-474 (2007)

[14] T. Tsao, M. Slamani, P. Varshney, D. Weiner, H. Schwarzlander, and S. Borek.: Ambiguity function for a bistatic radar. IEEE Transactions on Aerospace and Electronic Systems. vol. 33, no. 3, pp. 1041-1051 (1997)

[15] Gao C, Teh K C, Liu A.: Orthogonal Frequency Diversity Waveform with Range-Doppler Optimization for MIMO Radar. IEEE Signal Processing Letters. pp. 1201-1205 (2014) 